\title{
78. An Alternative Proof of a Generalized Principal Ideal Theorem.
}

By Tadao TANNAKa.

Mathematical Institute, Tohoku University, Sendai.

(Comm. by Z. Suetuna, M.J.A., Dec, 12, 1949.)

Recentry Mr. Terada1) has proved the following generalized principal theorem :

Theorem. Let $K$ be the absolute class field over $k$, and $\Omega$ a cycic intermediate field of $K / k$, then all the ambigous ideal classes of $\Omega$ will become principal in $K$.

I also generalized this theorem to the case of ray class field.2)

By using Artin's law of reciprocity we can state above theorem in terms of the Galois group, and we have

Theorem. Let $G$ be a metabelian group with commutator subgroup $G^{\prime}, H$ be an invariant subgroup of $G$ with the cyclic quotient group $G / H$, and $A$ element of $H$ with $A S A^{-1} S^{-1} \epsilon H^{\prime}$ ( $S$ being a generator of $G / H)$, then the "Verlagerung" $V(A)=\operatorname{II} T A \overline{T A}^{-1}$ from $H$ to $G^{\prime}$ is the unit element of $G$. Thereby $T$ runs over a fixed representative system of $G / H$, and $\overline{T A}$ means the representative corresponding to the coset $\overline{T A G^{\prime}}$.

At first we tried to solve this by means of Iyanaga's method depending upon Artin's splitting group, ${ }^{3)}$ which is generated by $G^{\prime}$ and the symbols $A_{\sigma}\left(A_{1}=1, \sigma \epsilon \Gamma=G / G^{\prime}\right)$, and with $\Gamma$ as operator system by rules

$$
\begin{gathered}
U^{\sigma}=S_{\sigma} U S_{\sigma}^{-1} \quad\left(U \varepsilon G^{\prime}\right), \\
A_{\tau}^{o}=A_{\sigma}^{-1} A_{\sigma \tau} D_{\sigma, \tau}^{-1},
\end{gathered}
$$

$S_{\sigma}$ being the representative of $G / G^{\prime}$ corresponding to $\sigma \varepsilon \Gamma$, and

$$
D_{\sigma, \tau}=S_{\sigma} S_{\tau} S_{\sigma \tau}^{-1} \text {. }
$$

But it seemed to us as if his method were not so easily applicable to our problem, and Terada at last checked the classical method of Furtwängler, ${ }^{4)}$ which brought him to success, after a rather complicated computation.

Here I will give a more simple proof, which depends upon Artin's splitting group. 
We first transform the problem into additive form (cf. Terada ${ }^{1)}$ ) and then by using Artin's splitting group (as in Tannaka ${ }^{2)}$ ) our theorem is reduced to a proposition concerning the additive group with a commutative ring $R$ as operator domain. Thus we now prove the

Theorem. Let $M$ be an additive group with the (not necessarily independent) base elements $c_{1}, c_{2}, \ldots, c_{22}$ and $c$, and

$$
N_{i} c_{i}=\sum_{r, s=1}^{n} A_{r, s}^{(i)} J_{j} c_{s}+\sum_{j=1}^{n} B_{j}^{(i)} \delta_{j} \quad(i=1,2, \ldots, n)
$$

where

$$
\begin{gathered}
\delta_{i}=\Delta c_{i}-\Delta_{i} c \\
N_{i} d_{i}=0 \\
A_{r, s}^{(1)}=-A_{s, r}^{(i)}, \quad A_{r, r}^{(i)}=0
\end{gathered}
$$

If then

$$
\sum \Gamma_{i} \delta_{i}=\sum F_{r, s} \Delta_{,} c_{s}\left(F_{r, s}=-F_{s, r}, F_{r, r}=0\right),
$$

we have

$$
N_{1} \ldots N_{n} \sum \Gamma_{i} c_{i}=0
$$

N.B. Concerning the meaning of notations we refer to Terada ${ }^{1)}$ and Tannaka ${ }^{2)} . \Delta$ represents the operator $1-S$, and $c$ is an element in $M$, which corresponds to $A_{S}$ in the splitting group. $\varepsilon_{i j}=\Delta_{j} c_{i}-\Delta_{i} c_{j}$ corresponds to the commutator of the form $S_{i} S_{j} S_{i}{ }^{-1} S_{j}{ }^{-1}$.

Proof. Form (1) and (2) we have

$$
\begin{gathered}
\sum_{j=1}^{n}\left(N_{i} \delta_{i j}-\sum_{r} A_{i j}^{(i)} \Delta_{r}-B_{j}^{(i)} d\right) c_{j}=-\sum_{j} B_{j}^{(i)} \Delta_{j} c, \\
\left(\delta_{i i}=1, \delta_{i j}=0, i \neq j ; i=1,2, \ldots, n\right),
\end{gathered}
$$

therefore by Cramér's method of elimination

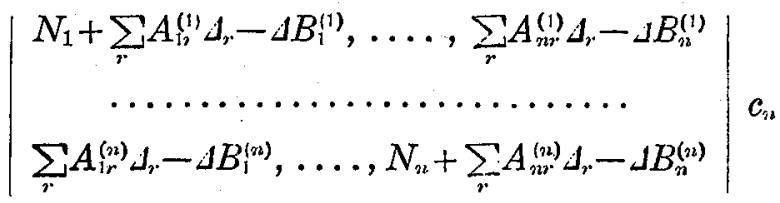

$$
\begin{aligned}
& =\left|\begin{array}{c}
N_{1}+\sum_{r} A_{1 r}^{(1)} d_{r}-\Delta B_{1}^{(1)}, \ldots \ldots,-\sum_{j} B_{j}^{(1)} d_{j} \\
\ldots \ldots \ldots \ldots \ldots \ldots \ldots \ldots \ldots \ldots \ldots, \sum_{j} B_{j}^{(2)} d_{j} \\
\sum_{r} A_{i r}^{(2)} \Delta_{r}-\Delta B_{l}^{(2)}, \ldots \ldots,
\end{array}\right| c,
\end{aligned}
$$


or

if we put

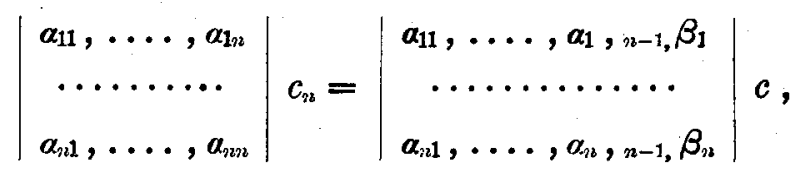

(9) . . $\quad\left\{\begin{array}{l}\alpha_{i j}=N_{i} \delta_{i j}+\sum_{r} A_{j r}^{(1)} \Delta_{r}-B_{j}^{(i)} \Delta, \\ \beta_{i}=-\sum_{j} B_{i}^{(i)} \Delta_{j} .\end{array}\right.$

We put further

$$
D=\left|a_{i j}\right|-N_{1} \ldots N_{p i}-
$$

In my preceding paper ${ }^{2)}$ I obtained the identity

$$
\left|\sum_{r} A_{j r}^{(i)} \Delta_{r}\right|=0
$$

and as its consequence

$$
\left|N_{i} \delta_{i j}+\sum_{r} A_{j r}^{(i)} \Delta_{r}\right|=N_{1} \ldots N_{n},
$$

so that in the expansion of $D$, every term has $\Delta$ as a faetor.

We now deduce the fundamental relation:

$$
N_{1} \ldots . N_{n} c_{i}=-\frac{D}{\Delta} \delta_{i}(i=1,2, \ldots, n) .
$$

In Terada's paper this formula is given in the expanded form, consequently it was somewhat complicated. Anyhow (13) was the key point of his success.

Without loss of generality we can restrict ourselves to the case $i=n$ in (13), so that we have only to prove

$$
N_{1} \ldots N_{n} c_{n z}=-\frac{D}{\Delta} \delta_{i 2} .
$$

From (8) and $\delta_{n}=\Delta c_{n}-\Delta_{n} c$ we have

$$
\begin{gathered}
N_{1} \ldots N_{n} c_{n}+D c_{n}=N_{1} \ldots N_{n} c_{n}+\frac{D}{d} \delta_{n}+\frac{D}{\Delta} \Delta_{n} c \\
=\left|\begin{array}{c}
\alpha_{11}, \ldots \alpha_{1},{ }_{n-1} \beta_{1} \\
\alpha_{n 21} \ldots \alpha_{n 2},{ }_{n-1} \beta_{n 2}
\end{array}\right| c
\end{gathered}
$$

so that $\left(13^{\prime}\right)$ may be reduced to

$$
\frac{D}{d} A_{23}=\left|\begin{array}{c}
\alpha_{11} \ldots \alpha_{1 n-1} \beta_{1} \\
\alpha_{21} \ldots \alpha_{n n-1} \beta_{n}
\end{array}\right|
$$


First we define $D_{n}$ by

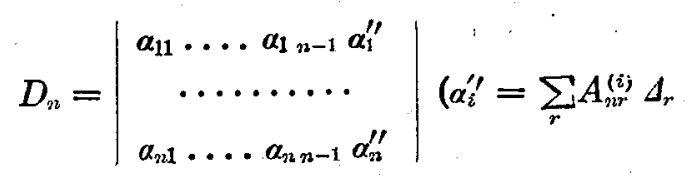

and prove

$$
\frac{D_{n b}}{\Delta} \Delta_{n}=\frac{D}{\Delta} \Delta_{n v}
$$

We have indeed

$$
\frac{D}{\Delta} \Delta_{n}-\frac{D_{n}}{\Delta} \Delta_{n s}=\left\{\left|\begin{array}{c}
\alpha_{11} \ldots \ldots \ldots \alpha_{1},{ }_{n-1} \\
\ldots \ldots \ldots \ldots \\
\alpha_{n-1}, 1 \ldots \ldots \alpha_{n-1},{ }_{n-1}
\end{array}\right| N_{n}-N_{1} \ldots N_{n}\right\} \frac{\Delta_{n s}}{\Delta}
$$

and as we have $A_{n} N_{n}=0$ and

$$
\begin{gathered}
\left|N_{i} \delta_{i j}+\sum_{r} A_{j n}^{(2)} \Delta_{r}\right|_{i, j \leq n-1} N_{n} \\
=\left|N_{i} \delta_{i j}+\sum_{r \leq n-1} A_{j r}^{(i)} A_{r}\right|_{i, j \leq n-1} N_{n}=\left(N_{1} \ldots N_{n-1}\right) N_{n}
\end{gathered}
$$

by (12). above expression reduces to 0 .

Now we prove the equality

$$
\frac{D_{n}}{4} \Delta_{n}=\left|\begin{array}{c}
\alpha_{11} \ldots \alpha_{1 n-1} \beta_{1} \\
\ldots \ldots \cdots \\
\alpha_{n 1} \ldots \alpha_{n n-1} \beta_{n n}
\end{array}\right|
$$

by induction on $n$.

If we expand both members of (17) in terms of $N_{i}$, and calling the relations $N_{i} d_{i}=0$ in mind, we see that the terms with $N_{i}$ as factor cancel out each other, for instance the terms with $N_{1}$ as. coefficient in the first member is

$$
\begin{aligned}
& N_{1}\left|N_{j} \delta_{i j}+\sum A_{j r}^{(i)} \Delta_{r}\right|_{i, j \geq 2} \frac{\Delta_{n}}{\Delta} \\
= & N_{1}\left|N_{j} \delta_{i j}+\sum_{r 2^{2}} A_{j r}^{(i)} \Delta_{r}\right|_{i, j \geq 2} \frac{\Delta_{n}}{\Delta}
\end{aligned}
$$

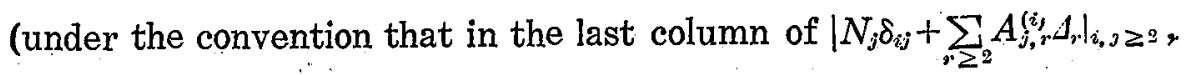
$N_{j} \delta_{i j}+\sum_{r=2} A_{j r}^{(i)} A_{r}$ should be replaced by $\left.\sum_{r, 2} A_{j r}^{(i)} A_{r}\right)$, so that the coefficient. of $N_{1}$ is of the same form as that of the first member of (17), except for the degree of determinant. So we have to prove only 


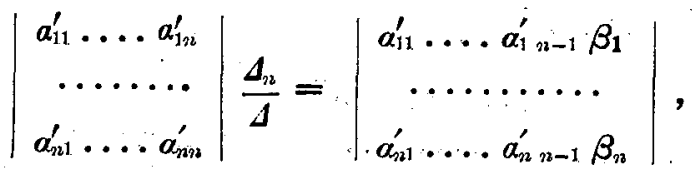

$$
\begin{aligned}
& \left(\alpha_{i j}^{\prime}=\sum_{r} A_{j r}^{(i)} A_{r}-B_{j}^{(i)} d, \beta_{i}=-\sum_{j} B_{r}^{(i)} d_{r}\right) .
\end{aligned}
$$

Expanding the both members, there remain only the terms with some $B_{3}^{(i)}$ as factor, for in the first member $\left|\sum_{r} A_{j r}^{(i)} \Delta_{r}\right|=0$ is the total contribution of such term. Hence we compare for instance the terms with $B_{1}^{(1)}$ as factor, and prove

$$
\begin{aligned}
& -B_{1}^{(1)} d_{n i}\left|\alpha_{i j}^{\prime}\right|_{k, j \geq 2}
\end{aligned}
$$

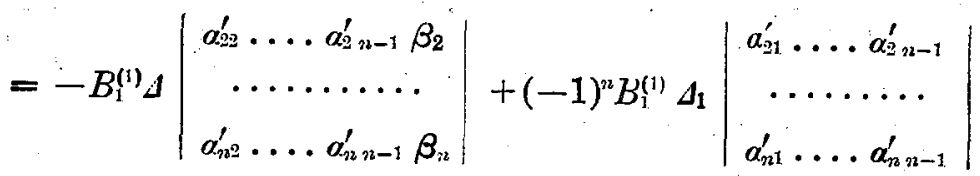

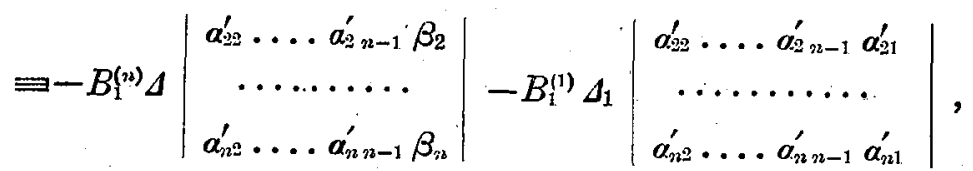

or

(19)

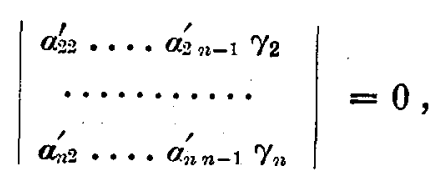

where

$$
\begin{aligned}
& \gamma_{i}=\alpha_{2 n}^{\prime} d_{i 2}-\Delta \beta_{i}+\alpha_{i 1}^{\prime} d_{1} \\
& =\left(\sum_{r} A_{2 n}^{(2)} \Delta_{r}-B_{n}^{(i)} d\right) d_{n}+\Delta \sum_{r} B_{r}^{(i)} d_{r}+\left(\sum_{r} A_{1 r}^{(i)} \Delta_{r}-B_{3}^{(i)} d\right) d_{1} \\
& =\sum_{s=1,2} \sum_{r} A_{s r}^{(i)} \Delta_{s} A_{r}+A_{1} \sum_{r<\lambda^{2}} B_{r}^{(2)} d_{r} \text {. }
\end{aligned}
$$

But as

$$
a_{i 2}^{\prime}+\cdots+a_{i, j,-1}^{\prime}+\gamma_{i}=\sum_{s, r} A_{s r}^{(i)} \Delta_{s} \Delta_{r}=0
$$

we have established (19), and the proof of (16) is completed.

We now proceed to the second part of our proof, that is, the proof of (6).

As it holds $N_{1} \ldots N_{n} \sum \Gamma_{i} c_{i}=\sum \Gamma_{i} N_{1} \ldots N_{n} c_{i}=-\sum \Gamma_{i} \frac{D}{d} \delta_{i}$ by (13) and

$$
=-\frac{D}{J} \sum F_{r, s} u_{r} c_{s}
$$


by (5), it suffices to prove for instance the equality

$$
\frac{D}{d} \varepsilon_{12}=\frac{D}{J}\left(d_{2} c_{1}-\Delta_{1} c_{2}\right)=0
$$

But it follows from (16) and (17)

$$
\begin{aligned}
& \frac{D}{J} \varepsilon_{12}=\frac{D_{2}}{\Delta} \Delta_{2} c_{1}-\frac{D_{1}}{J} \Delta_{1} c_{\varepsilon} \\
& =\left|\begin{array}{c}
\alpha_{11} \beta_{1} \alpha_{13} \ldots \ldots \alpha_{12} \\
\ldots \ldots \ldots \ldots \\
\alpha_{211} \beta_{n} \alpha_{n 3} \ldots \ldots \\
\ldots
\end{array}\right| c_{n n}-\left|\begin{array}{c}
\beta_{1} \alpha_{12} \ldots \ldots \alpha_{1 n 2} \\
\ldots \ldots \ldots \\
\beta_{n 2} \alpha_{n 2} \ldots \ldots \alpha_{n n 2}
\end{array}\right| c_{2} \\
& =\left|\begin{array}{c}
\alpha_{11} c_{1}+\alpha_{12} c_{2}, \beta_{1}, \alpha_{13}, \ldots, \alpha_{1 i n} \\
\ldots \ldots \ldots, \ldots, \ldots, \\
a_{n 1} c_{1}+\alpha_{n 2} c_{2}, \beta_{n}, \alpha_{233}, \ldots, \alpha_{22 n}
\end{array}\right| \\
& =\left|\begin{array}{c}
\sum \alpha_{1 r} c_{r}+\beta_{1} c, \beta_{1}, \alpha_{13}, \ldots \ldots \alpha_{1 n} \\
\ldots \ldots \ldots \ldots \ldots \ldots \ldots \\
\sum \alpha_{n 2 r} c_{r}+\beta_{s 2} c, \beta_{22}, \alpha_{23}, \ldots \ldots \alpha_{2 n z}
\end{array}\right| \text {, }
\end{aligned}
$$

and as $\sum_{r} \alpha_{i r} c_{r}+\beta_{i} c=0$ by (14), we have $\frac{D}{\Delta} \varepsilon_{12}=0$ as desired, q.e.d.

\section{References}

1) F. Terada: On the generalization of the principal ideal theorem, Tôhoku Math. J., (2) 1, No. 2 (1949).

2) T. Tannaka: Some remarks concerning principal ideal theorem, Tôhoku Math. J., (2) 1, No. 2 (1949).

3) S. Iyanaga: Zum Beweis des Hauptidealsatzes, Hamb. Abh., 10 (1934).

4) Ph. Furtwängler: Beweis des Hauptidealsatzes, Hamb. Abh, 7 (1930).

Concerning 1) and 2) we also refer to the previous note, titled "A generalization of principal ideal theorem" in Proe. Acad. Tokyo (1949). 